\title{
Laser-induced bubbles create valves and pumps
}

\begin{abstract}
Scientists in China have investigated how laser-induced bubbles could be used as optically controlled valves and pumps in microfluidic circuitry and lab-on-a-chip systems. Kai Zhang and co-workers from Hong Kong Polytechnic University and Jinan University report how the beam from a 5 W, $980 \mathrm{~nm}$ fibre-pigtailed diode laser can heat chromium pads on the internal walls of a transparent, water-filled polymer microchannel and thus create localized bubbles for blocking or regulating flow (Lab Chip 11, 1389-1395; 2011).

The researchers used these microvalves to control the rate at which two aqueous solutions - water, and water containing red ink - were mixed in the output of a Y-shaped microchannel circuit. They also demonstrated that heating a tear-shaped metallic pad produces a pumping effect; the bubble grows faster at the head of the tear than at the base, thus forcing the fluid along the channel at a speed that can be controlled by varying the laser power. The researchers report velocities in the range of $100-400 \mu \mathrm{m} \mathrm{s}^{-1}$, which is equivalent to flow rates of 7.2$28.8 \mu \mathrm{l} \mathrm{h}{ }^{-1}$ for microchannels measuring $500 \mu \mathrm{m} \times 40 \mu \mathrm{m}$ in cross-section.
\end{abstract}
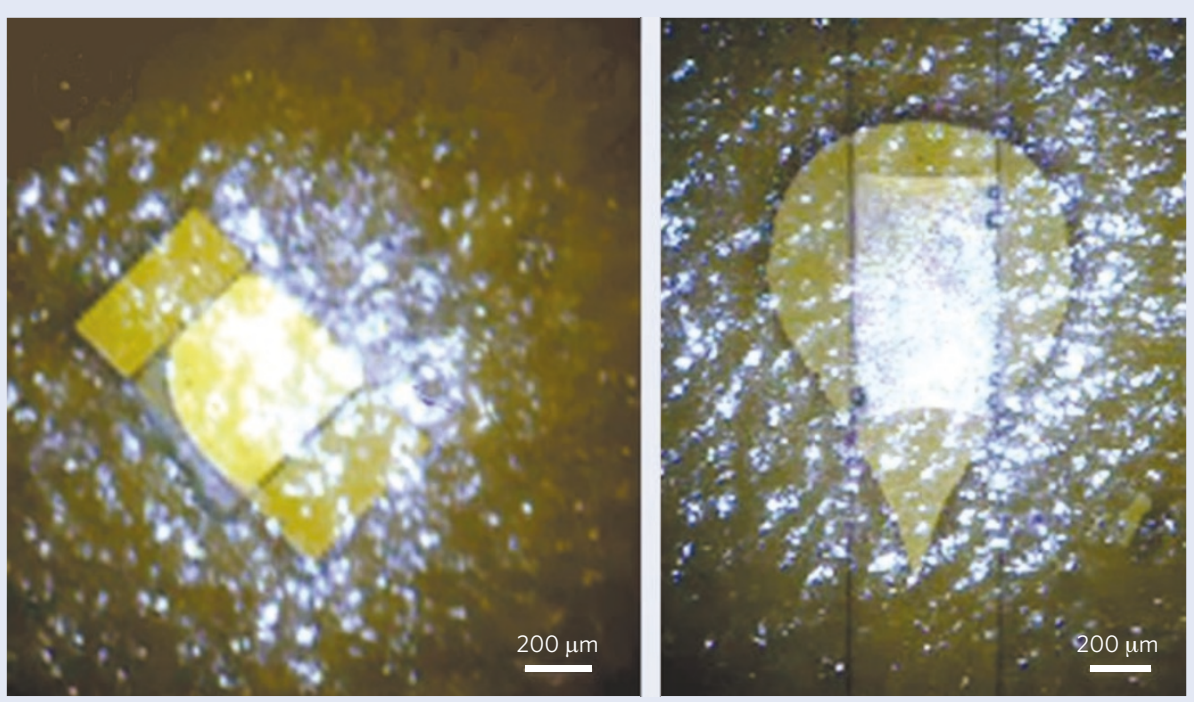

"The phenomenal success of thermal bubbles in ink-jet printing has stimulated broad interest in bubble technology, particularly in the booming areas of microelectromechanical systems and lab-on-a-chip technology," comment Zhang and co-workers in their paper. "The response time in our demonstrations are typically one to tens of seconds, which is slow for most applications. This is because the channels are quite wide $(500 \mu \mathrm{m})$ and the laser power is kept low for the convenience of fabrication and experiments. Much faster speeds can be achieved with narrower channels and higher power lasers."

OLIVER GRAYDON

\section{Photons in a diamond microring}

Researchers have significantly enhanced the single-photon emission of atomic defects in single-crystal diamond by coupling the photons to microring resonators - a technique that may represent a powerful addition to the integrated quantum photonics toolkit.

\section{Brian R. Patton and Jeremy L. O'Brien}

$(2)$ uantum technologies aim to gain unprecedented power and functionality in information processing, secure communication and precision measurement by harnessing quantum superposition and entanglement. Of the physical systems being used to develop these quantum technologies, photons - both as quantum bits (qubits) and as brokering agents for qubits embodied in other systems - are particularly appealing for their lownoise, high-speed transmission and ease of manipulation at the single-photon level ${ }^{1}$.
Photons are indispensable for quantum communication and quantum metrology, and are a leading approach towards the long-term goal of quantum computing ${ }^{2}$.

Reporting in Nature Photonics, Andrei Faraon and colleagues at HewlettPackard Laboratories in Palo Alto, California, USA, have now shown that single photons emitted by point defects in diamond can be coupled to microring resonators fabricated from a single crystal of diamond ${ }^{3}$. The many superlative qualities of diamond, such as its exceptional hardness, thermal conductivity and wide range of optically active defects, make it attractive for use in integrated optical devices. However, these same properties also introduce challenges when processing the material. The work of Faraon et al. opens the way for the integration of diamond into quantum photonics devices, enabling a host of applications in quantum technologies as well as optical signal processing and sensing.

Integrated quantum photonics aims to bring together all the key components of photonic quantum technologies. There has already been much progress in the 